\title{
Two-Point Correlators of Fermionic Currents in External Magnetic Field
}

\author{
Alexandra Dobrynina ${ }^{1, \star}$, Ilya Karabanov ${ }^{1, \star \star}$, Alexander Parkhomenko ${ }^{1, \star \star \star}$, and Lubov \\ Vassilevskaya ${ }^{2}$ \\ ${ }^{1}$ P. G. Demidov Yaroslavl State University, Yaroslavl, Russia \\ ${ }^{2}$ Fulda University of Applied Sciences, Fulda, Germany
}

\begin{abstract}
We study the two-point correlation functions under an influence of the constant homogeneous magnetic field. In addition to the correlators of scalar, pseudoscalar, vector and axial-vector fermionic currents, we calculate the non-diagonal one including the tensor and pseudoscalar currents. The tensor current is a fermionic part of the Pauli Lagrangian relevant for the electromagnetic interaction of fermions through the anomalous magnetic moment. Its contribution to the photon polarization operator is briefly discussed.
\end{abstract}

\section{Introduction}

The Standard Model — the gauge theory based on the $S U(3)_{C} \otimes S U(2)_{L} \otimes U(1)_{Y}$ group — is intensively testing for its consistency and no significant experimental deviations from theoretical predictions have been found yet [1]. More important, many experiments are quite sensitive to vacuum effects which are usually calculated as radiative corrections to observables within the perturbation theory. In particular, to get a good agreement with experimental data at the Large Hadron Collider (LHC), production cross-sections should be known in the Next-to-Leading Order (NLO) and some of them even in the Next-to-Next-to-Leading Order (NNLO). Moreover, vacuum effects can also generate rare processes which are forbidden at the tree level in the Standard Model. Good examples of such processes are the radiative $H \rightarrow \gamma \gamma$ and gluonic $H \rightarrow g g$ Higgs decays, already observed at the LHC [1].

In a difference to LHC, electron-positron colliders allow to get detail information about the photon propagation from the energy scan in the electron-positron annihilation process $e^{-} e^{+} \rightarrow$ hadrons. In this case, one needs to know the photon polarization function (see, for example, [2, 3]):

$$
3 Q^{2} \Pi\left(Q^{2}\right)=i \int d^{4} x e^{i(q x)}\left\langle 0\left|T\left\{j^{\mu}(x) j_{\mu}(0)\right\}\right| 0\right\rangle
$$

where $j_{\mu}(x)=\sum_{f} \bar{\psi}_{f}(x) \gamma_{\mu} \psi_{f}(x)$ is the flavor-singlet quark vector current and $Q^{2}=-q^{2}$. The Feynman diagram describing the $\gamma \rightarrow \gamma$ transition via virtual charged fermions in the one loop approximation is presented in Fig. 1. To get the vacuum average in (1), one needs to put $y^{\mu}=0$. The func-

\footnotetext{
^e-mail: dobrynina@uniyar.ac.ru

$\star \star$ e-mail: karabanovilya@ rambler.ru

$\star \star \star$ e-mail: parkh@uniyar.ac.ru
} 


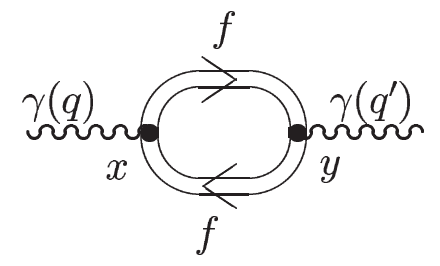

Figure 1. Feynman diagram describing the $\gamma \rightarrow \gamma$ transition via virtual fermions. Double lines indicate that effects of an external electromagnetic field are taken into account exactly in the fermion propagators.

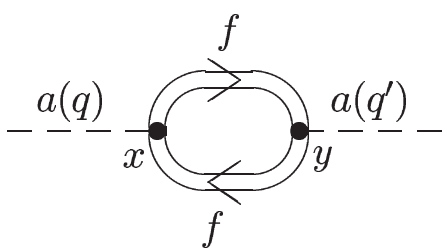

Figure 2. Feynman diagram describing the $a \rightarrow a$ transition via virtual fermions. Double lines indicate that effects of an external electromagnetic field are taken into account exactly in the fermion propagators.

tion $\Pi\left(Q^{2}\right)$ can be related with a similar two-point correlation function (the photon polarization function) $\Pi^{\mathrm{em}}\left(Q^{2}\right)[2,3]$ originated by the quark electromagnetic current $j_{\mu}^{\mathrm{em}}(x)=\sum_{f} q_{f} \bar{\psi}_{f}(x) \gamma_{\mu} \psi_{f}(x)$, where $q_{f}$ is the electric charge of the quark. Many physical observables are connected with $\Pi^{\mathrm{em}}\left(Q^{2}\right)$ (see, for example, [4]), in particular, the cross-section ratio is proportional to the imaginary part of $\Pi^{\mathrm{em}}\left(Q^{2}\right)$ as follows:

$$
R(s) \equiv \frac{\sigma\left(e^{-} e^{+} \rightarrow \text { hadrons }\right)}{\sigma\left(e^{-} e^{+} \rightarrow \mu^{-} \mu^{+}\right)}=12 \pi \operatorname{Im} \Pi^{\mathrm{em}}(-s-i \epsilon)
$$

In addition to the vector-current correlator, massless correlators of scalar and tensor quark currents at higher orders in $\alpha_{s}$ were also calculated recently [5].

For more complicated physical systems like astrophysical objects and the early Universe or in heavy-ion collisions one needs to take into account effects of an external medium. Note that such a medium can significantly modify a wave-function and dispersion properties of a charged fermion and, as a consequence, change substantially the quantum field of the photon. The Feynman diagram describing the $\gamma \rightarrow \gamma$ transition via virtual charged fermions is presented in Fig. 1, where double lines indicate that fermion propagators are modified by an external electromagnetic field and plasma. Detail discussions of the photon polarization operator under external conditions can be found in the books $[6,7]$ and references therein. In what follows, we neglect the plasma effects on the fermion propagator and restrict ourselves by accounting effects of the background electromagnetic field only.

The matrix element of the $a \rightarrow a$ transition in an external electromagnetic field background also exemplifies the two-point correlation function of two axial-vector currents. The corresponding Feynman graph is shown in Fig. 2. This diagram determines the electromagnetic correction to the axion mass squared $m_{a}^{2}$ [8-10]. The effective neutrino-photon interaction, $\bar{v} v \gamma$-vertex, in an external electromagnetic field [7] is the other example of the two-point correlations which, in addition to the diagonal vector correlator responsible for the photon polarization operator and diagonal axial-vector correlator, includes the non-diagonal correlator of vector and axial-vector electron currents.

The general case of the two-point correlator modified by the constant homogeneous magnetic field was studied in Ref. [11]. The Lagrangian density of a local fermion interaction with a generalized current $J_{A}(x)$ which can be, for example, a photon field, neutrino current, derivative of the axion field and etc. (with the corresponding constants), has the following form [7, 11]:

$$
\mathcal{L}_{\text {int }}(x)=\left[\bar{f}(x) \Gamma^{A} f(x)\right] J_{A}(x),
$$

where $f(x)$ is the quantum fermion field and $\Gamma_{A}$ is any of the $\gamma$-matrices from the standard set $\left\{1, \gamma_{5}, \gamma_{\mu}, \gamma_{\mu} \gamma_{5}\right\}$ [12]. The two-point correlation function based on the fermionic currents of the form 
in (3) can be presented as follows [7, 11]:

$$
\Pi_{A B}=\int d^{4} X \mathrm{e}^{-i(q X)} \operatorname{Sp}\left\{S_{\mathrm{F}}(-X) \Gamma_{A} S_{\mathrm{F}}(X) \Gamma_{B}\right\}
$$

where $S_{\mathrm{F}}(X)$ is the Lorentz-invariant part of an exact fermion propagator calculated in an external field background [13]. We assume that the external field is equal to the constant homogeneous magnetic field. Among the existing representations of $S_{\mathrm{F}}(X)$ for this field, we accept the so-called Fock-Schwinger one $[13,14]$ in which the fermion propagator has the explicitly Lorentz covariant form. Correlations among the scalar, pseudoscalar, vector and axial-vector fermionic currents in the Fock-Schwinger formalism were already studied [7, 11] but correlators of the tensor fermionic current $J_{\mu v}(x) \equiv\left[\bar{f}(x) \sigma_{\mu \nu} f(x)\right]$ with the other currents $J_{A}(x)$ introduced above, under an influence of the magnetic field was not considered in this approach, on the best of our knowledges.

In this paper, we present the propagator of a charged fermion in the constant homogeneous magnetic field in the Fock-Schwinger representation, show some selected results for the two-point correlation functions and conclude with a discussion of further applications of the formalism considered.

\section{Propagator in Constant Homogeneous Magnetic Field}

The equation for the fermion propagator in the electromagnetic field is as follows [13]:

$$
\left[i \hat{\partial}-e Q_{f} \hat{A}(x)-m_{f}\right] G_{\mathrm{F}}(x, y)=\delta^{(4)}(x-y),
$$

where $Q_{f}$ and $m_{f}$ are the relative charge and mass of the fermion, $\hat{\partial}=\partial_{\mu} \gamma^{\mu}$, and $\hat{A}=A_{\mu} \gamma^{\mu}$ with $A^{\mu}(x)$ being the external-field four-potential. The general representation of the charged fermion propagator in the Fock-Schwinger representation is known well $[13,14]$ :

$$
G_{\mathrm{F}}(x, y)=\mathrm{e}^{i \Omega(x, y)} S_{\mathrm{F}}(x-y),
$$

where the Lorentz non-invariant phase $\Omega(x, y)$ can be written in the form [13, 14]:

$$
\Omega(x, y)=-e Q_{f} \int_{y}^{x} d \xi^{\mu}\left[A_{\mu}(\xi)+\frac{1}{2} F_{\mu \nu}(\xi-y)^{v}\right] .
$$

Here $F_{\mu \nu}$ is the strength tensor of the external electromagnetic field. In the two-point correlation function, the phase factors of the two propagators cancel each other:

$$
\Omega(x, y)+\Omega(y, x)=0,
$$

and the Lorentz-invariant part of the fermion propagator is required only [see Eq. (4)].

Let us consider a pure constant homogeneous magnetic field, $\mathbf{B}=(0,0, B)$. The corresponding four-potential can be written in the exactly Lorentz-covariant form, $A_{\mu}(x)=-F_{\mu \nu} x^{\nu} / 2$. Minkowski space filled with the constant homogeneous magnetic field is divided into two subspaces: the Euclidean one with the metric tensor $\Lambda_{\mu v}=(\varphi \varphi)_{\mu \nu}$ which is nothing else but the plane orthogonal to the field direction, and pseudo-Euclidean one with the metric tensor $\tilde{\Lambda}_{\mu \nu}=(\tilde{\varphi} \tilde{\varphi})_{\mu \nu}$. The metric tensor of Minkowski space can be written as a difference of the subspace metrics, $g_{\mu \nu}=\tilde{\Lambda}_{\mu \nu}-\Lambda_{\mu v}$. In each subspace there are also the Levi-Civita symbols $\varphi_{\mu \nu}$ and $\tilde{\varphi}_{\mu v}$ which are the dimensionless tensor of the external magnetic field and its dual:

$$
\varphi_{\mu \nu}=\frac{F_{\mu v}}{B}, \quad \tilde{\varphi}_{\mu v}=\frac{1}{2} \varepsilon_{\mu \nu \rho \sigma} \varphi^{\rho \sigma},
$$


where $\varepsilon_{\mu \nu \rho \sigma}$ is the antisymmetric Levi-Civita symbol with the definition $\varepsilon^{0123}=1$ [12]. An arbitrary four-vector $a^{\mu}=\left(a_{0}, a_{1}, a_{2}, a_{3}\right)$ can be decomposed into the two orthogonal components:

$$
a_{\mu}=\tilde{\Lambda}_{\mu \nu} a^{\nu}-\Lambda_{\mu \nu} a^{v}=a_{\| \mu}-a_{\perp \mu} .
$$

For the scalar product of two four-vectors one has

$$
(a b)=(a b)_{\|}-(a b)_{\perp},
$$

where the terms on r.h.s. are non-trivial in one subspace only, i.e.:

$$
(a b)_{\|}=(a \tilde{\Lambda} b)=a^{\mu} \tilde{\Lambda}_{\mu \nu} b^{\nu}, \quad(a b)_{\perp}=(a \Lambda b)=a^{\mu} \Lambda_{\mu \nu} b^{v} .
$$

After these notations are introduced, we can write the Lorentz-invariant part of the fermion propagator as follows [7]:

$$
\begin{aligned}
S_{\mathrm{F}}(X) & =-\frac{i \beta}{2(4 \pi)^{2}} \int_{0}^{\infty} \frac{d s}{s^{2}}\left\{(X \widetilde{\Lambda} \gamma) \cot (\beta s)-i(X \widetilde{\varphi} \gamma) \gamma_{5}\right. \\
& \left.-\frac{\beta s}{\sin ^{2}(\beta s)}(X \Lambda \gamma)+m_{f} s[2 \cot (\beta s)+(\gamma \varphi \gamma)]\right\} \\
& \times \exp \left(-i\left[m_{f}^{2} s+\frac{1}{4 s}(X \tilde{\Lambda} X)-\frac{\beta \cot (\beta s)}{4}(X \Lambda X)\right]\right),
\end{aligned}
$$

where $\beta=e B\left|Q_{f}\right|$. Note that Eq. (4) is written in terms of this part of the charged fermion propagator.

\section{Natural Orthogonal Basis in Magnetic Field Background}

Correlators having rank different from zero, are convenient to decompose in some set of four independent vectors. In the magnetic field background, such an orthogonal basis naturally exists [7]:

$$
\begin{aligned}
& b_{\mu}^{(1)}=(q \varphi)_{\mu}, \quad b_{\mu}^{(2)}=(q \tilde{\varphi})_{\mu} \\
& b_{\mu}^{(3)}=q^{2}(\Lambda q)_{\mu}-(q \Lambda q) q_{\mu}, \quad b_{\mu}^{(4)}=q_{\mu},
\end{aligned}
$$

and an arbitrary four-vector $a_{\mu}$ can be presented as follows:

$$
a_{\mu}=\sum_{i=1}^{4} a_{i} \frac{b_{\mu}^{(i)}}{\left(b^{(i)} b^{(i)}\right)}, \quad a_{i}=a^{\mu} b_{\mu}^{(i)}=\left(a b^{(i)}\right) .
$$

The norms of the vectors (14) can be calculated easily:

$$
\begin{aligned}
& \left(b^{(1)} b^{(1)}\right)=-(q \Lambda q), \quad\left(b^{(2)} b^{(2)}\right)=-(q \tilde{\Lambda} q), \\
& \left(b^{(3)} b^{(3)}\right)=-q^{2}(q \tilde{\Lambda} q)(q \Lambda q), \quad\left(b^{(4)} b^{(4)}\right)=q^{2} .
\end{aligned}
$$

An arbitrary second-rank tensor $T_{\mu \nu}$ can be decomposed similarly:

$$
T_{\mu \nu}=\sum_{i, j=1}^{4} T_{i j} \frac{b_{\mu}^{(i)} b_{v}^{(j)}}{\left(b^{(i)} b^{(i)}\right)\left(b^{(j)} b^{(j)}\right)}, \quad T_{i j}=b_{\mu}^{(i)} T^{\mu v} b_{v}^{(j)}=\left(b^{(i)} T b^{(j)}\right) .
$$

This procedure can be extended to higher rank tensors.

As all the technical details are already discussed, let us present several examples of the two-point correlators. 


\section{Examples of Correlators}

Here, we present several examples of two-point correlation functions.

\subsection{Photon Polarization Operator}

The photon polarization operator is the typical example of the two-point correlation function.

The Lagrangian density of the fermion-photon interaction in QED is well-known $[12,15]$ :

$$
\mathcal{L}_{\mathrm{QED}}(x)=\sum_{f} e Q_{f}\left[\bar{f}(x) \gamma_{\mu} f(x)\right] A^{\mu}(x)
$$

where $f(x)$ and $A^{\mu}(x)$ are quantized fermion and photon fields, respectively, and $Q_{F}$ is the electric charge of the fermion in units of the elementary charge, $e>0$. Comparison of (18) with the generalized Lagrangian (3) shows that the generalized current is $J_{\mu}(x)=e Q_{f} A_{\mu}(x)$.

In the momentum space, the matrix element of the $\gamma \rightarrow \gamma$ transition induced by the Lagrangian (18) can be written in the form $[12,15]$ :

$$
i M_{\gamma \rightarrow \gamma}=\varepsilon_{\mu}^{*}(q) \Pi^{\mu v}(q) \varepsilon_{\nu}(q),
$$

where $\varepsilon_{\mu}(q)$ is the polarization vector of the photon with the momentum $q^{\mu}$. The Lorentz tensor $\Pi^{\mu \nu}(q)$ is the photon polarization operator, very well studied within QED both in vacuum $[12,15]$ and under an influence of an active media $[6,7]$.

\subsection{Axion Self-Energy}

The other example of the two-point correlation function is the axion self-energy. In the magnetic field, it was calculated in Refs. [8-10].

The Lagrangian density of the fermion-axion interaction is as follows [6]:

$$
\mathcal{L}_{a f}(x)=\sum_{f} \frac{g_{a f}}{2 m_{f}}\left[\bar{f}(x) \gamma^{\mu} \gamma_{5} f(x)\right] \partial_{\mu} a(x),
$$

where $f(x)$ and $a(x)$ are quantum fermion and axion fields, respectively, $\partial_{\mu} a(x)=\partial a(x) / \partial x^{\mu}, g_{a f}=$ $C_{f} m_{f} / f_{a}$ is the dimensionless Yukawa constant, $C_{f}$ is the dimensionless factor dependent on the axion model [6], and $\gamma_{5}=-i \gamma^{0} \gamma^{1} \gamma^{2} \gamma^{3}$ [12]. This Lagrangian is also of the type (3) with the generalized current $J_{\mu}(x)=\left(g_{a f} / 2 m_{f}\right) \partial_{\mu} a(x)$.

The $a \rightarrow a$ transition amplitude, $M_{a \rightarrow a}$, calculated in the momentum space determines the shift of the axion mass squared due to the vacuum effects [10]:

$$
M_{a \rightarrow a}=-\delta m_{a}^{2} .
$$

The corresponding diagram is shown in Fig. 2 where double solid lines indicate that effects of the external magnetic field on the fermion propagator are taken into account exactly. So, one can see that $M_{a \rightarrow a}$ is the two-point correlation function determined by the time-ordered product of two axialvector fermionic currents. Our calculations confirm the results presented in Ref. [10]:

$$
\begin{aligned}
M_{a \rightarrow a}\left(q^{2}, q_{\perp}^{2}, \beta\right) & =\sum_{f} \frac{g_{a f}^{2} \beta}{8 \pi^{2}} \int_{0}^{\infty} \frac{d t}{\sin (\beta t)} \int_{0}^{1} d u\left[q_{\|}^{2} \cos (\beta t)-q_{\perp}^{2} \cos (\beta t u)\right] \times \\
& \times \exp \left\{-i\left[m_{f}^{2} t-\frac{q_{\|}^{2}}{4} t\left(1-u^{2}\right)+q_{\perp}^{2} \frac{\cos (\beta t u)-\cos (\beta t)}{2 \beta \sin (\beta t)}\right]\right\},
\end{aligned}
$$


where $q^{2}=q_{\|}^{2}-q_{\perp}^{2}$. Note that two proper-time variables $s_{1}$ and $s_{2}$ entering each of the propagators have been replaced by the other two: $t=s_{1}+s_{2}$ and $u=\left(s_{1}-s_{2}\right) / t$.

The invariant matrix element (22) contains both pure vacuum and field-induced parts and the field-induced contribution $\Delta M\left(q^{2}, q_{\perp}^{2}, \beta\right)$ to $M_{a \rightarrow a}$ is as follows:

$$
\Delta M\left(q^{2}, q_{\perp}^{2}, \beta\right)=M_{a \rightarrow a}\left(q^{2}, q_{\perp}^{2}, \beta\right)-M_{a \rightarrow a}\left(q^{2}, 0,0\right) .
$$

Note that this quantity is free from the ultraviolet divergences [10].

\subsection{Correlator of Pseudoscalar and Tensor Currents}

The non-diagonal correlator of pseudoscalar and tensor currents is the second-rank tensor. Taking into account its antisymmetry under permutations, from six independent coefficients in the basis decomposition (17), three only are non-trivial:

$$
\begin{aligned}
\Pi_{12}^{(\mathrm{PT})}\left(q^{2}, q_{\perp}^{2}, \beta\right) & =\frac{\beta}{8 \pi^{2}} q_{\|}^{2} q_{\perp}^{2} \int_{0}^{\infty} d t \int_{0}^{1} d u \frac{\sin (\beta t u)}{\sin (\beta t)}[\cot (\beta t)-u \cot (\beta t u)] \\
& \times \exp \left\{-i\left[m_{f}^{2} t-\frac{q_{\|}^{2}}{4} t\left(1-u^{2}\right)+q_{\perp}^{2} \frac{\cos (\beta t u)-\cos (\beta t)}{2 \beta \sin (\beta t)}\right]\right\}, \\
\Pi_{23}^{(\mathrm{PT})}\left(q^{2}, q_{\perp}^{2}, \beta\right) & =-\frac{\beta}{8 \pi^{2}} q_{\|}^{2} \int_{0}^{\infty} \frac{d t}{t \sin ^{2}(\beta t)} \int_{0}^{1} d u\left\{4 \sin ^{2}(\beta t)\left[m_{f}^{2} t+i\right]\right. \\
& \left.+q_{\|}^{2} t\left[2 \cos (\beta t) \cos (\beta t u)+2 u \sin (\beta t) \sin (\beta t u)+\left(1-u^{2}\right) \sin ^{2}(\beta t)-2\right]\right\} \\
& \times \exp \left\{-i\left[m_{f}^{2} t-\frac{q_{\|}^{2}}{4} t\left(1-u^{2}\right)+q_{\perp}^{2} \frac{\cos (\beta t u)-\cos (\beta t)}{2 \beta \sin (\beta t)}\right]\right\}, \\
\Pi_{24}^{(\mathrm{PT})}\left(q^{2}, q_{\perp}^{2}, \beta\right) & =\frac{\beta}{32 \pi^{2}} q_{\|}^{2} q_{\perp}^{2} \int_{0}^{\infty} \frac{d t}{t \sin ^{2}(\beta t)} \int_{0}^{1} d u\left\{4 \sin { }^{2}(\beta t)\left[m_{f}^{2} t+i\right]\right. \\
& \left.+q_{\|}^{2} t\left(1-u^{2}\right) \sin ^{2}(\beta t)-2 q_{\perp}^{2} t[\cos (\beta t) \cos (\beta t u)+u \sin (\beta t) \sin (\beta t u)-1]\right\} \\
& \times \exp \left\{-i\left[m_{f}^{2} t-\frac{q_{\|}^{2}}{4} t\left(1-u^{2}\right)+q_{\perp}^{2} \frac{\cos (\beta t u)-\cos (\beta t)}{2 \beta \sin (\beta t)}\right]\right\} .
\end{aligned}
$$

The coefficients $\Pi_{21}^{(\mathrm{PT})}\left(q^{2}, q_{\perp}^{2}, \beta\right), \Pi_{32}^{(\mathrm{PT})}\left(q^{2}, q_{\perp}^{2}, \beta\right)$, and $\Pi_{42}^{(\mathrm{PT})}\left(q^{2}, q_{\perp}^{2}, \beta\right)$ differ by the sign from $\Pi_{12}^{(\mathrm{PT})}\left(q^{2}, q_{\perp}^{2}, \beta\right), \Pi_{23}^{(\mathrm{PT})}\left(q^{2}, q_{\perp}^{2}, \beta\right)$, and $\Pi_{24}^{(\mathrm{PT})}\left(q^{2}, q_{\perp}^{2}, \beta\right)$ because of the tensor anti-symmetry.

Correlators of other generalized currents with the tensor one will be presented elsewhere [16].

\section{Applications of Correlators}

As discussed in Subsec. 4.1, the standard polarization operator is related with the correlator of two vector currents. Models beyond the Standard Model can effectively modify the QED Lagrangian with an extra term called the Pauli Lagrangian density [17-19]:

$$
\mathcal{L}_{\mathrm{AMM}}(x)=\frac{\mu_{f}}{4}\left[\bar{f}(x) \sigma_{\mu v} f(x)\right] F^{\mu v}(x),
$$


where $\mu_{f}$ is the anomalous magnetic moment (AMM) of the fermion. Note that this term is gaugeand Lorentz-invariant but non-renormalizable within the modified QED. The existence of (24) in the effective QED Lagrangian should also change the photon polarization operator. A contribution linear in the fermion AMM is related with the correlator of the vector and tensor fermionic currents. Its influence on the photon properties requires a detail discussion and will be presented elsewhere [16].

The strong-field limit can be also important as the resulting expressions are simplified drastically and allow detail understanding of results without cumbersome numerical analysis. It is worth to note the strong-magnetic-field formalism suggested by Loskutov and Skobelev [20,21] which allow to perform calculations similar to the conventional ones developed for vacuum. Unfortunately, some loop diagrams are uncertain and a clear procedure is required to fix them. Let us mentioned in connection with this the analysis of the axion self-energy in the magnetic field [8-10]. The correct result was obtained only after the strong-field limit was found explicitly [10].

The technique we presented here can be extended for calculations of three-point correlation functions. The well-known example of such a correlator is the three-photon vertex in the external constant homogeneous magnetic field calculated by Adler [22]. Two of us have already an experience of calculating the three-point correlation functions connected with the effective $a \gamma \gamma$-vertex of the axion interacting with two photons in the crossed [23, 24] and magnetic [25] field configurations of the external background. Note that the same vertex calculated in the crossed field later by Skobelev [26] differs from ours but a reason still remains unclear. One should also mentioned the recent analysis of the magnetic-field influence on the radiative Higgs decay [27]. Some other three-point vertices, like the one describing an interaction of neutrinos with two photons, are also of special importance for astrophysics $[6,7]$.

\section{Conclusions}

Two-point correlators in presence of a constant homogeneous external magnetic field are considered. This analysis extended the previous one [11] by including the fermionic tensor current into consideration. With new correlators, modifications of the photon polarization operator induced by the Pauli Lagrangian density can be studied. The computer technique developed for two-point correlators is planned to be applied for three-point correlation functions.

\section{Acknowledgements}

The paper is partially supported by the Russian Foundation for Basic Research (Project No. 1502-06033-a) and by the YSU Initiative Scientific Research Activity (Project No. AAAA-A16116070610023-3). A. D. acknowledges financial support by the Russian Foundation for Basic Research (Project No. 16-32-00066 мол-а) and by the Dynasty Foundation.

\section{References}

[1] C. Patrignani et al. (Particle Data Group), Chin. Phys. C40, 100001 (2016)

[2] P.A. Baikov, K.G. Chetyrkin, J.H. Kuhn, J. Rittinger, JHEP 07, 017 (2012), 1206.1284

[3] P.A. Baikov, K.G. Chetyrkin, J.H. Kuhn, J. Rittinger, Phys. Lett. B714, 62 (2012), 1206.1288

[4] K. Chetyrkin, J. Kuehn, PoS LL2016, 047 (2016), 1607 . 08374

[5] K.G. Chetyrkin, A. Maier, Nucl. Phys. B844, 266 (2011), 1010. 1145

[6] G.G. Raffelt, Stars as Laboratories for Fundamental Physics (University of Chicago Press, Chicago \& London, 1996) 
[7] A.V. Kuznetsov, N.V. Mikheev, Electroweak Processes in External Active Media, Vol. 252 of Springer Tracts in Modern Physics (Springer-Verlag, New York, 2013)

[8] V.V. Skobelev, Phys. Atom. Nucl. 61, 2123 (1998), [Yad. Fiz. 61, 2236 (1998)]

[9] A.V. Borisov, P.E. Sizin, J. Exp. Theor. Phys. 88, 1 (1999), [Zh. Eksp. Teor. Fiz. 115, 3 (1999)], hep-ph/9904356

[10] L.A. Vasilevskaya, N.V. Mikheev, A.Ya. Parkhomenko, Phys. Atom. Nucl. 64, 294 (2001), [Yad. Fiz. 64, 342 (2001)]

[11] M.Yu. Borovkov, A.V. Kuznetsov, N.V. Mikheev, Phys. Atom. Nucl. 62, 1601 (1999), [Yad. Fiz. 62, 1714 (1999)]

[12] M.E. Peskin, D.V. Schroeder, An Introduction to Quantum Field Theory (Addison-Wesley, Reading, Massachusetts, 1995)

[13] C. Itzykson, J.B. Zuber, Quantum Field Theory (McGraw-Hill, New York, 1980)

[14] J.S. Schwinger, Phys. Rev. 82, 664 (1951)

[15] V.B. Berestetsky, E.M. Lifshitz, L.P. Pitaevsky, Course of Theoretical Physics. Vol. 4. Quantum Electrodynamics (Pergamon, Oxford, 1982)

[16] L.A. Vasilevskaya, A.A. Dobrynina, I.V. Karabanov, A.Ya. Parkhomenko, in preparation (2017)

[17] P.M. Lavrov, J. Phys. A18, 3455 (1985)

[18] H.K. Lee, Y. Yoon, JHEP 03, 086 (2007), hep-th/0611134

[19] A. Barducci, R. Giachetti, G. Pettini, Eur. Phys. J. C70, 445 (2010), 1006. 3871

[20] V.V. Skobelev, Russ. Phys. J. 18, 1481 (1975)

[21] Y.M. Loskutov, V.V. Skobelev, Phys. Lett. A56, 151 (1976)

[22] S.L. Adler, Annals Phys. 67, 599 (1971)

[23] L.A. Vasilevskaya, N.V. Mikheev, A.Ya. Parkhomenko, Phys. Atom. Nucl. 60, 2041 (1997), [Yad. Fiz. 60, 2224 (1997)]

[24] N.V. Mikheev, A.Ya. Parkhomenko, L.A. Vassilevskaya, Phys. Rev. D60, 035001 (1999), hep-ph/9903415

[25] N.V. Mikheev, A.Ya. Parkhomenko, L.A. Vasilevskaya, Phys. Atom. Nucl. 63, 1046 (2000), [Yad. Fiz. 63, 1122 (2000)]

[26] V.V. Skobelev, J. Exp. Theor. Phys. 88, 209 (1999), [Zh. Eksp. Teor. Fiz. 115, 385 (1999)]

[27] N.K. Nielsen, Phys. Rev. D90, 016010 (2014), 1312 . 6280 